\title{
ELECTRIC POWER COMPENSATION OF THE LARGE SCALE ACCELERATOR USING SMES ${ }^{\#}$
}

\author{
H. Sato ${ }^{1}$, T. Shintomi ${ }^{2}$, M. Shirakata, KEK, Ibaraki, Japan \\ T. Ise, Y. Miura, Osaka University, Suita, Japan \\ S. Nomura, Integrated Research Institute, Tokyo Tech, Tokyo, Japan
}

\begin{abstract}
Power supply for the large scale accelerator magnets draws a large amount of power from the utility network. For example, the pulse power amplitude and the dissipation power of J-PARC $50 \mathrm{GeV}$ synchrotron magnet power are estimated to be about $170 \mathrm{MW}$ and $30 \mathrm{MW}$, respectively. Superconducting Magnetic Energy Storage (SMES) is one of candidates to compensate these large pulse load and line voltage fluctuation. Study on circuit configuration of the power supply with SMES has been continued. Present status of the R \& D for the SMES system and the laboratory scale experiment results will be discussed.
\end{abstract}

\section{INTRODUCTION}

The accelerator complex called J-PARC (Japan Proton Accelerator Research Complex) with a joint project between KEK (High Energy Accelerator Research Organization) and JAEA (Japan Atomic Energy Agency) is under construction at the Tokai campus of JAEA to be commissioned in 2007 (2008 for main ring)[1]. J-PARC is a research-facility complex covering a variety of research programs. The complex in the final stage will be composed of a 400-MeV normal-conducting linac and a $600-\mathrm{MeV}$ superconducting linac to increase the energy from 400 to $600 \mathrm{MeV}^{*}$, a $3-\mathrm{GeV}$ rapid cycling synchrotron (RCS), which provides proton beams at $333 \mu \mathrm{A}(1 \mathrm{MW})$, and a $50-\mathrm{GeV}$ synchrotron ring, which provides proton beams at $15 \mu \mathrm{A}(0.75 \mathrm{MW})$. The RCS will be used for the materials and life science experiment with muon and neutron sources and also used as a booster synchrotron for the $50-\mathrm{GeV}$ main ring (MR). The MR will be operated with the repetition rate of around $0.3 \mathrm{~Hz}$ in order to extract a proton beam for the nuclear and particle physics experiments and neutrino experiment. The project is processing with two steps, Phase-I and Phase-II. Although the main ring will be operated at 40 $\mathrm{GeV}$ or $30 \mathrm{GeV}$ in phase-I, it will be upgraded to $50 \mathrm{GeV}$ in Phase-II. The demanded pulse electric power at the 50 $\mathrm{GeV}$ operation will be very huge and this large pulse power will disturb an electric power network. Therefore some means is needed to equip for the pulse power compensation.

\# Work supported by Joint Development Research at KEK.

1 hikaru.sato@kek.jp

2 Advanced Research Institute for the Sciences and Humanities

Nihon University.

* In Phase-1, an $181 \mathrm{MeV}$ linac is under operation for a $400 \mathrm{MeV}$. linac.

\section{MR POWER SUPPLY AND ELECTRIC POWER DEMAND}

The MR has a three-super period configuration with a family of 96 bending magnets and eleven families of 216 quadrupole magnets. The bending magnets are divided into three sections and six groups in total. Each group is excited with a unit of power supply. Therefore, the power supply for the bending magnets is composed of six power supply units, while each family of the quadrupole magnets has one power supply unit $[2,3]$.

The pulse electric power is required to excite the magnets corresponding to the resistive and inductive parts at the $50 \mathrm{GeV}$ operation. Since the power supply adopts the self-commutated converter system utilizing IGBT or IEGT, little reactive power is generated and power factor is about unity.

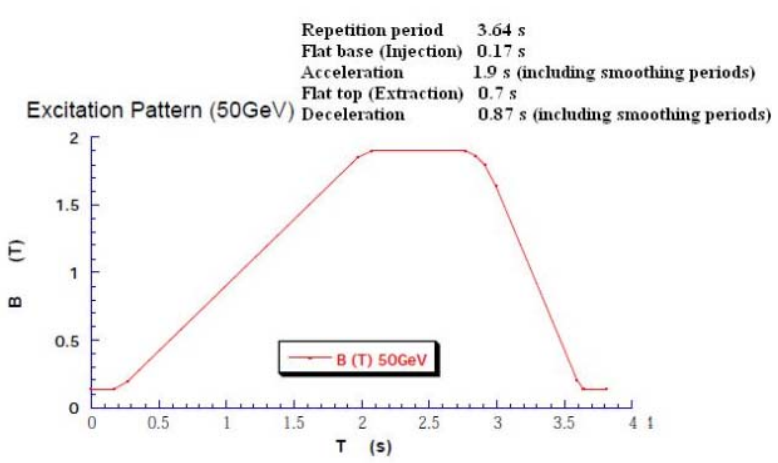

Fig. 1 Typical excitations for the MR magnets at the 50 GeV operation

One of the typical excitations for the MR magnets at the $50 \mathrm{GeV}$ operation is that after the injection period of 0.17 seconds, the magnetic field is linearly increased up to $2 \mathrm{~T}$ at the flat top (FT) in $1.9 \mathrm{~s}$, kept for $0.7 \mathrm{~s}$ at FT, and decreased to the initial value for the next step of beam acceleration in $0.87 \mathrm{~s}$. The total cycle time is $3.64 \mathrm{~s}$ as shown in Fig. 1. The peak power is generated at the end of the full excitation and the beginning of the deexcitation of the magnets. The peak powers at each timing are $+105 \mathrm{MW}$ and $-65 \mathrm{MW}$, respectively, and the total pulse power amplitude is $170 \mathrm{MW}$. The big pulse power swing affects a connected network with disturbances over a permissible level. In order to reduce such disturbances, 
SMES could be very useful. The original design indicates that the disturbance level is allowable if the total pulse power amplitude is less than 100MW (Simulation results by Tokyo Electric Power Company). Three cases of the electric power demand are shown in Fig. 2.

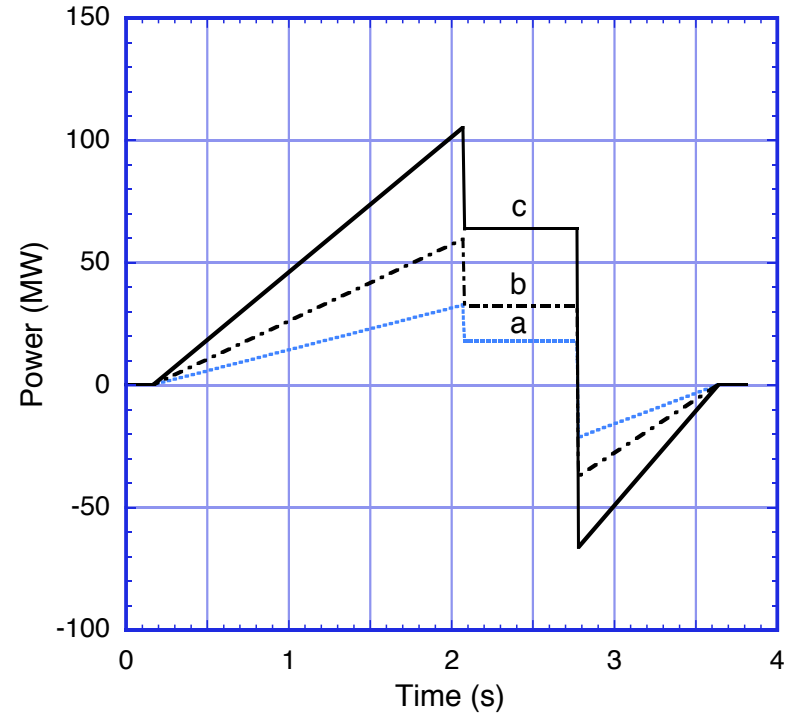

Fig. 2. The active power of the MR magnets for the various operation patterns of $3.64 \mathrm{~s}$ repetition. a: $30 \mathrm{GeV}$, b: $40 \mathrm{GeV}$ and c: $50 \mathrm{GeV}$.

\section{SMES FOR PULSE POWER COMPENSATOR}

For the original design of the energy storage system, a doubly-fed flywheel generator (FWG) system has been under consideration as shown in Fig.3 [2, 3] and a few research studies were performed [4,5]. At this present, we propose a SMES system for the other solution for the pulse power compensation. In case of the SMES, the system can be installed at an ac line as like as the FWG system as shown in Fig. 4, and also at a dc line of the power supply for the MR magnets as shown in Fig. 5.

As mentioned above, we assume that the pulse power over that at the $40 \mathrm{GeV}$ operation has to be compensated by the SMES system and the compensation power is estimated as around $21 \mathrm{MJ}$. If $30 \%$ of the stored energy in the SMES system is assumed to be used with a conservative estimate, the SMES system, which has the capacity of $70 \mathrm{MJ}$, is necessary. We have designed two types of SMES systems. One is a simple type installed at an ac line as like as the FWG system. We propose the other excellent system configuration that six SMES units are connected at the dc side of the power supply.

For the case of ac link, SMES will be installed at the proposed site of the FWG system, but there is a difficulty of installation site of refrigerator system for the case of $\mathrm{dc}$ link.

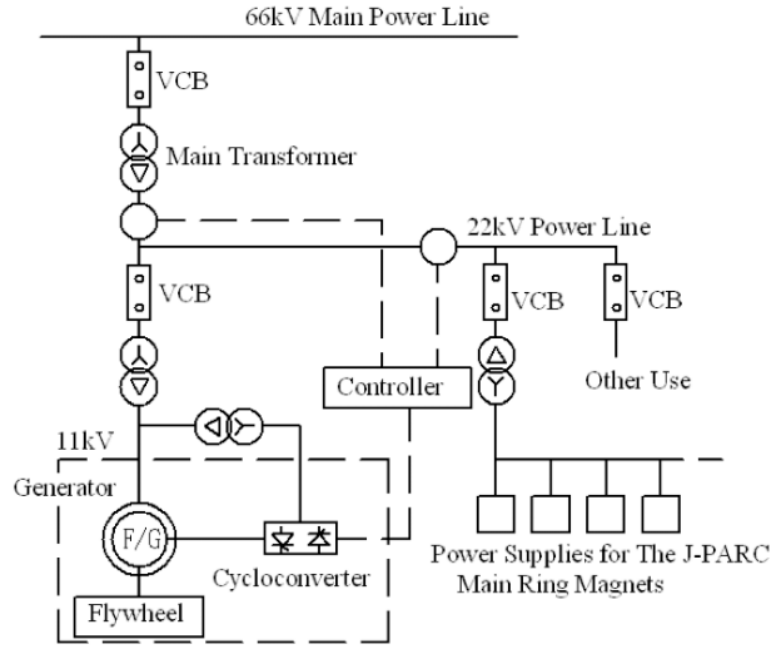

Fig.3 Conceptual block diagram of the power supply system with FWG for the $50-\mathrm{GeV}$ MR.

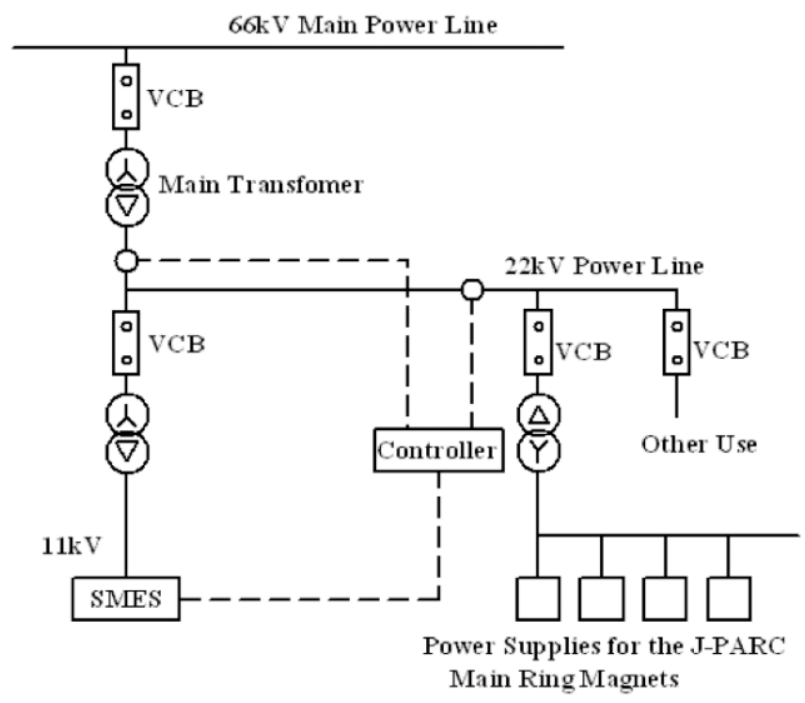

Fig.4 Conceptual block diagram of the power supply system with SMES at an ac line.

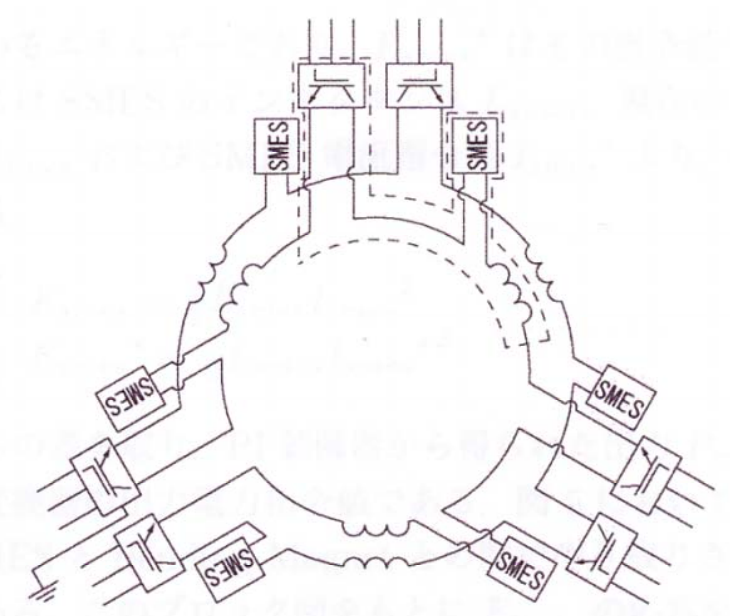

Fig.5 Proposed distributed SMES system configuration of dc link. 


\section{DESIGN OF DISTRIBUTED SMES COIL UNIT}

In consideration of the six units power supply, superconducting coil for SMES is also composed of six units. A four solenoid coils configuration with opposite polarities is proposed as shown in Fig. 6, although we proposed the Force-Balanced Coil SMES formerly [6]. At the design, the parameters were fixed as that the maximum magnetic flux density was $5.0 \mathrm{~T}$ with the Rutherford type $\mathrm{Nb}$-Ti conductor and the height of coil was twice the diameter. The stored energy of one coil is 2.0 MJ and 5.0 MJ, respectively. On the cases of 2.0 MJ and 5.0 MJ, the total stored energies by four pole coils and six units are $48 \mathrm{MJ}$ and $120 \mathrm{MJ}$, respectively. In order to compensate the pulse power, $21 \mathrm{MJ}$ should be charged and discharged, then, $44 \%$ and $18 \%$ of the stored energy are used, respectively. Specifications of the coil unit are shown in Table 1.

For the Nb-Ti conductor, it is better to use the conductor, which has been developed for the LHC main dipole and quadrupole magnets at CERN [7], since it's good performance and mass-production. There are two types of conductors for the inner layer coil and the outer layer coil of the main dipole. We use the LHC inner layer coil cable, since the cross section of wire is large and the load factor is safer. The conductor is composed of 28 strands with a diameter of $1.065 \mathrm{~mm}$ and the critical current is $30 \mathrm{kA}$ at $5 \mathrm{~T}$ and $4.2 \mathrm{~K}$.

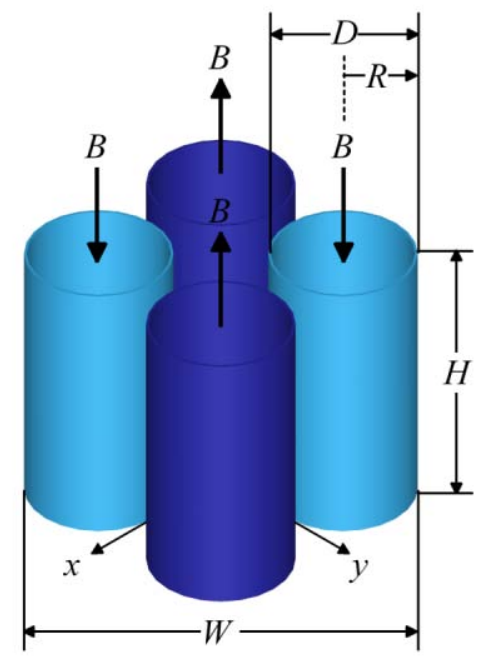

Fig. 6 The four solenoid coils configuration.

\section{EXPERIMENTAL STUDY}

Experimental study for the dc link connection of SMES by laboratory scale model with 1/50 scale for the voltage and $1 / 100$ scale for the current is being carried out [8]. The preliminary results show that initial starting-up of the system and power compensation by SMES can be successfully performed, although response characteristics should be improved through improvements of the experimental controller. We will continue this experiment to investigate more detailed knowledge and present in near future.

Table 1: SEMS Coil Parameter

\begin{tabular}{|lll|}
\hline Stored Energy of Each Coil(MJ) & 2.0 & 5.0 \\
Maximum Magnetic Flux Density(T) & 5.0 & 5.0 \\
Coil Current (kA) & 10 & 10 \\
Average Diameter of Coil (m) & 0.51 & 0.69 \\
Height of Coil (m) & 0.924 & 1.407 \\
Number of Turn & 440 & 603 \\
Magnetomotive Force (MAT) & 4.4 & 6.0 \\
5G Line Radius of 4 Pole (m) & 3 & 4 \\
Total Stored Energy of 6 Units (MJ) & 48 & 120 \\
Radius of 6 Units SMES (m) & 9 & 12 \\
\hline
\end{tabular}

\section{CONCLUSION}

A $50 \mathrm{GeV}$ operation of the J-PARC MR in phase-II requires some compensation device to reduce unallowable disturbances to a power network. Typical pattern of the $50 \mathrm{GeV}$ operation such as pattern c in Fig. 2 induce the power swing $+105 \mathrm{MW}$ and $-65 \mathrm{MW}$ peak-to-peak. The SMES system connected at the dc side of the power supply has been proposed and considered as an excellent solution for the power compensator. Six coil distributed SMES system was proposed and typical coil design was discussed. Experimental study by the laboratory scale model has been performed. We will continue this experiment to investigate more detailed knowledge and present in near future. Further, we will study the effect of the leakage magnetic field from SMES and ac loss of the conductor so far. Smoothing the power rating with using SMES will be also effective for the compact accelerators such as the medical use to reduce the running cost.

\section{REFERENCES}

[1] http://j-parc.jp/en/AccSci.html

[2] M. Muto et al., Proceedings of the APAC'98, KEK, Japan, 1998, p.384.

[3] Accelerator Technical Design Report for J-PARC, KEK Report 2002-13.

[4] H. Akagi et al., Proceedings of the PAC'99, New York, 1999, p.3749.

[5[ H. Akagi and H. Sato, IEEE Transactions on Power Electronics, 17(2002)109.

[6] T. Shintomi, et al., IEEE Transaction on Applied Superconductivity, Vol.16, No.2, June 2006, p.628.

[7] LHC Design Report, vol. 1; LHC Main Ring (CERN 2004-003-v1).

[8] S. Yamamoto, Master Thesis, Osaka University Graduate School, February 2007, in Japanese. 